\title{
Picosecond Photoinduced Reflectivity Studies of GaN Prepared by Lateral Epitaxial Overgrowth
}

\author{
M. Wraback ${ }^{1}$, H. Shen ${ }^{1}$, C.J. Eiting ${ }^{2{ }^{*}}$, J.C. Carrano ${ }^{2 * *}$, and R.D. Dupuis ${ }^{2}$ \\ ${ }^{1}$ U.S. Army Research Laboratory, Sensors and Electron Devices Directorate, 2800 \\ Powder Mill Road, Adelphi, MD 20783, USA \\ ${ }^{2}$ Microelectronics Research Center, Department of Electrical Engineering, University of \\ Texas at Austin, Austin, TX 78712-1100, USA \\ *current address: ${ }^{*}$ Photonics Research Center, Department of Electrical Engineering and \\ Computer Science, U.S. Military Academy, West Point, NY 10996, USA; ${ }^{\dagger}$ WPAFB, OH \\ 45433, USA
}

\begin{abstract}
The pump-probe technique has been used to perform room temperature studies of the photoinduced changes in the reflectivity $\Delta \mathrm{R}$ associated with exciton and carrier dynamics in $\mathrm{GaN}$ prepared by lateral epitaxial overgrowth. For resonant excitation of cold excitons, the $\Delta \mathrm{R}$ decay possesses a 720 ps component attributed to the free exciton lifetime in this high quality material. For electrons with small excess energy $(<50 \mathrm{meV})$, the strong increase in the $\Delta \mathrm{R}$ decay rate with decreasing excitation density suggests that screening of the Coulomb interaction may play an important role in the processes of carrier relaxation and exciton formation. The faster decay times at a given carrier density observed for hot (> $100 \mathrm{meV}$ ) electron relaxation are attributed to electron-hole scattering in conjunction with the screened electron-LO phonon interaction.
\end{abstract}

\section{INTRODUCTION}

Gallium Nitride $(\mathrm{GaN})$ has become an important material for ultraviolet light emitters and detectors, as well as high power, high frequency electronic devices. It has been demonstrated that growth of GaN on sapphire by lateral epitaxial overgrowth (LEO) [1-3] greatly reduces the threading dislocation density in this material. A device lifetime of more than $10000 \mathrm{~h}$ at room temperature has been reported for $\mathrm{cw}$ operation of InGaN multiple quantum well laser diodes employing LEO GaN grown by metalorganic chemical vapor deposition (MOCVD) [1], and a significant decrease of p-n junction reverse leakage current in LEO materials has also been observed [3]. However, little is known about the room temperature dynamics of resonantly created excitons and free carriers with low excess energy crucial to an understanding of device performance in this high quality GaN.

In this paper we present a time-resolved pump-probe study of exciton and free carrier dynamics in LEO GaN in which photoinduced changes in reflectivity $\Delta \mathrm{R}$ are monitored on a picosecond timescale. The $\Delta \mathrm{R}$ transients were obtained as a function of excitation intensity for three cases: (i) resonantly created excitons; (ii) electrons with low excess energy; and (iii) electrons with sufficient excess energy to emit longitudinal optical (LO) phonons. The data obtained from these measurements provides information about exciton lifetimes in this high quality material, the dynamics of exciton formation and screening in the presence of cold electron-hole pairs, and the cooling of a hot electron distribution due to carrier-carrier scattering and the partially screened electron-LO phonon interaction. 


\section{EXPERIMENTAL CONSIDERATIONS}

The sample employed in this study was a $\sim 5 \mu \mathrm{m}$ thick undoped GaN LEO film on an undoped $\mathrm{GaN} /$ sapphire substrate. $15 \mu \mathrm{m}$-wide $\mathrm{SiO}_{2}$ stripes deposited by plasma enhanced chemical vapor deposition (PECVD) were patterned with $3 \mu \mathrm{m}$ windows between the stripes. The growth of the LEO GaN upon this material was accomplished by rotating-disk MOCVD at a growth pressure of 100 torr and a growth temperature of $1070^{\circ} \mathrm{C}$.

Visible/near infrared laser pulses derived from the signal beam of a $250 \mathrm{kHz}$ Ti:sapphire regenerative amplifier-pumped optical parametric amplifier were compressed to less than 100 fs and frequency doubled to obtain a tunable source of ultraviolet pulses for frequency degenerate pump and probe measurements. The temporal evolution of the pump-induced change in the reflectivity of the probe pulse was monitored for various excitation photon energies and intensities. For excitation of free carriers using photon energies near the bandgap of $\mathrm{GaN}, \Delta \mathrm{R}$ is essentially proportional to the change in the real part of the dielectric function $\Delta \varepsilon_{1}$, which may be obtained from the spectral dependence of the change in the imaginary part of the dielectric function $\Delta \varepsilon_{2}$ by means of the Kramers-Kronig relation

$$
\Delta \varepsilon_{1}(\omega)=\frac{2}{\pi} \int_{0}^{\infty} \frac{\omega^{\prime} \Delta \varepsilon_{2}\left(\omega^{\prime}\right) d \omega^{\prime}}{\omega^{\prime 2}-\omega^{2}},
$$

where $\Delta \varepsilon_{2}$ is a measure of the photoinduced bleaching associated with the absorption saturation of band-to-band transitions [4]. The basic insight of this experiment is that while photoinduced transmission measurements primarily probe $\Delta \varepsilon_{2}$, only providing information about the sum of the electron and hole distribution functions at energies $\mathrm{E}_{\mathrm{e}}$ and $\mathrm{E}_{\mathrm{h}}$ defined by the probe frequency and the band structure [4], photoinduced reflectivity measurements are indicative of the integrated behavior of the carrier distribution functions. For $\mathrm{GaN}$ the electron effective mass $\left(\sim 0.2 \mathrm{~m}_{\mathrm{o}}\right)$ is much less than the hole effective mass $\left(\sim 2 \mathrm{~m}_{\mathrm{o}}\right)$ [5]. Since the ratio of the electron and hole excess energies is inversely proportional to the ratio of their effective masses in the parabolic band approximation, it follows that the electrons receive almost all of the excess energy from the excitation pulse. Moreover, the density of states is proportional to $\mathrm{m}^{3 / 2}$, implying that the density of states near $\mathbf{k}=0$ in the conduction band is much smaller than that in the valence band. Therefore, for a given density of electron-hole pairs, the contribution of the electron distribution to the photoinduced bleaching is much larger than that of the hole distribution. These observations suggest that our time-resolved photoinduced reflectivity measurements primarily probe the electron dynamics. For resonant excitation of excitons, one may discuss the changes in the real and imaginary parts of the dielectric function in terms of the bleaching of the exciton peak [6].

\section{EXPERIMENTAL RESULTS}

Figures 1,2, and 3 show $\Delta \mathrm{R}$ as a function of time delay for pulses with center wavelengths of $364.5 \mathrm{~nm}, 357.5 \mathrm{~nm}$ and $347 \mathrm{~nm}$, respectively. In all cases a positive pulse-width-limited rise in $\Delta \mathrm{R}$ is observed at zero time delay. For excitation at $364.5 \mathrm{~nm}$, the pump creates cold $\mathrm{A}(\mathrm{B})$ excitons, as determined from the $\mathrm{cw}$ luminescence spectrum.

F99W11.55 


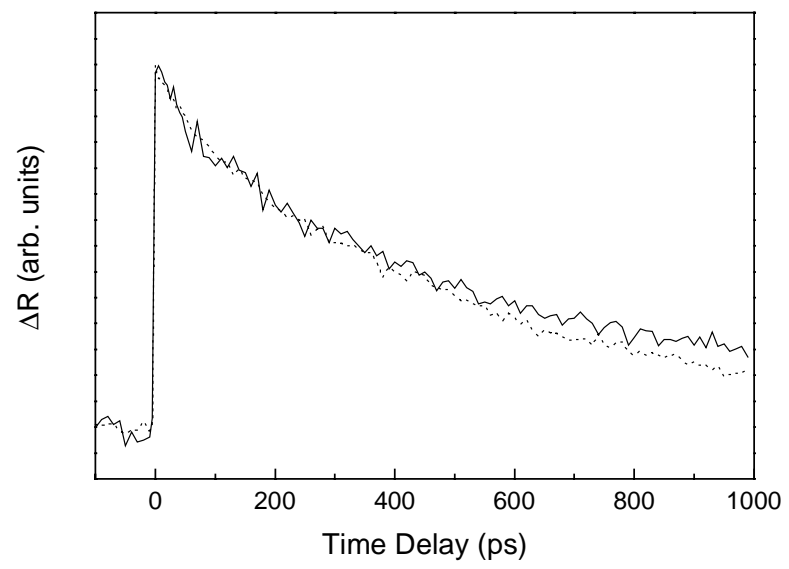

Figure 1 Normalized change in probe reflectivity $\Delta \mathrm{R}$ as a function of time delay between pump and probe pulses with center wavelength $\lambda=364.5 \mathrm{~nm}$. Solid line: carrier density $\mathrm{n}=4.4 \times 10^{18} \mathrm{~cm}^{-3}$; dotted line: $\mathrm{n}=4 \times 10^{19} \mathrm{~cm}^{-3}$.

In this case the $\Delta \mathrm{R}$ decay at low pump intensity (solid line) is well described by a biexponential decay of the form $\Delta \mathrm{R}(\mathrm{t})=\mathrm{C}_{1} \exp \left(-\mathrm{t} / \tau_{1}\right)+\mathrm{C}_{2} \exp \left(-\mathrm{t} / \tau_{2}\right)$, where $\mathrm{t}$ is the time delay between the pump and probe pulses and $\mathrm{C}_{1}, \mathrm{C}_{2}, \tau_{1}$, and $\tau_{2}$ are fitting parameters.

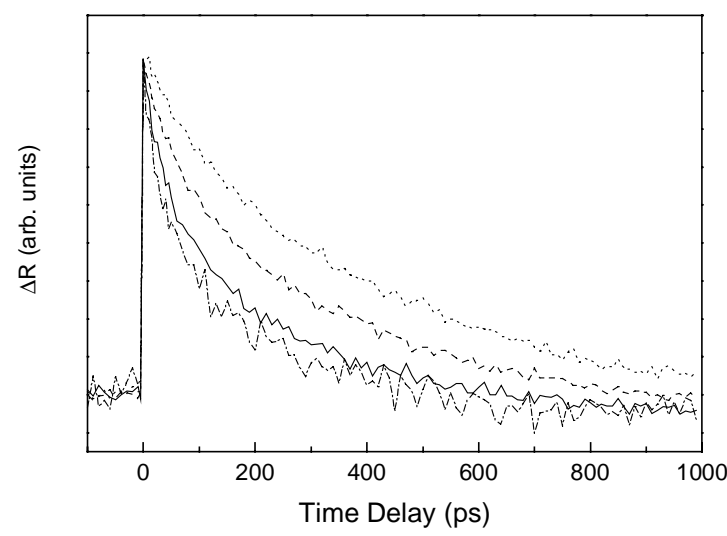

Figure 2 Normalized change in probe reflectivity $\Delta \mathrm{R}$ as a function of time delay between pump and probe pulses with center wavelength $\lambda=357.5 \mathrm{~nm}$. Dotted line: carrier density $\mathrm{n}=2 \times 10^{19} \mathrm{~cm}^{-3}$; dashed line: $\mathrm{n}=1 \times 10^{19} \mathrm{~cm}^{-3}$; solid line: $\mathrm{n}=3.3 \times 10^{18} \mathrm{~cm}^{-3}$; dash-dotted line: $\mathrm{n}=1.3 \times 10^{18} \mathrm{~cm}^{-3}$. 
The best fit is obtained for $\tau_{1} \sim 55 \mathrm{ps}, \tau_{2} \sim 720 \mathrm{ps}$, and $\mathrm{C}_{2} / \mathrm{C}_{1} \sim 4$. These numerical values remain about the same for lower pump intensities. When the pump intensity is raised by nearly an order of magnitude (dotted line) the fast decay remains the same, while the slow decay becomes slightly faster $\left(\tau_{2} \sim 550 \mathrm{ps}\right)$.

For excitation at $357.5 \mathrm{~nm}$, the electrons possess less than $50 \mathrm{meV}$ of excess energy above the conduction band edge. In this case the $\Delta \mathrm{R}$ decays become faster as the carrier density is lowered. Using the time at which $\Delta \mathrm{R}$ reaches half its maximum value as a measure of the decay time $\tau_{\mathrm{hm}}$, we find that $\tau_{\mathrm{hm}}$ is about 5 times shorter $(\sim 46 \mathrm{ps})$ for the lowest carrier density data shown in figure 2 (dash-dotted line) than for the highest density data ( $226 \mathrm{ps}$, dotted line).

Figure 3 shows $\Delta \mathrm{R}$ data for excitation at $347 \mathrm{~nm}$. In this case the initial excess energy of the electron distribution is greater than $100 \mathrm{meV}$, exceeding the LO phonon energy of $92 \mathrm{meV}$. As observed in figure 2, the $\Delta \mathrm{R}$ decay becomes faster with decreasing excitation density, with $\tau_{\mathrm{hm}}$ dropping by nearly a factor of 2 , from $67 \mathrm{ps}$ to $35 \mathrm{ps}$, when the carrier density is lowered by nearly an order of magnitude. The inset of figure 3 shows that the curve obtained for the lower carrier density in the main figure possesses an initial rapid decay with a 1.25 ps time constant. Upon reducing the carrier density by an additional factor of 2 , this initial decay disappears (dotted line, inset).

\section{DISCUSSION}

For resonant excitation of $\mathrm{A}(\mathrm{B})$ excitons, photoinduced bleaching of the excitonic

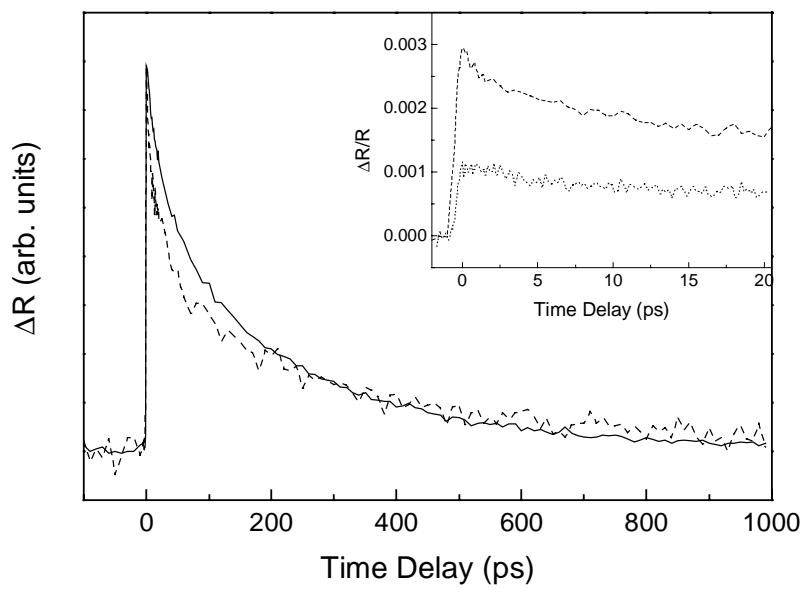

Figure 3 Normalized change in probe reflectivity $\Delta \mathrm{R}$ as a function of time delay between pump and probe pulses with center wavelength $\lambda=347 \mathrm{~nm}$. Solid line: carrier density $\mathrm{n}=4 \times 10^{19} \mathrm{~cm}^{-3}$; dashed line: $\mathrm{n}=4.5 \times 10^{18} \mathrm{~cm}^{-3}$. Inset: $\Delta R / R$ at short time delays for carrier densities of $\mathrm{n}=4.5 \times 10^{18} \mathrm{~cm}^{-3}$ (dashed line) and $\mathrm{n}=2.2 \times 10^{18} \mathrm{~cm}^{-3}$ (dotted line). 
absorption creates a primarily positive $\Delta \mathrm{R}[6]$. The decay of this $\Delta \mathrm{R}$ can be associated with the removal of free excitons by recombination or trapping at deep levels. The fact that the slow decay in figure 1 becomes only slightly faster when the intensity is increased by about an order of magnitude implies that the decay of $\Delta \mathrm{R}$ for our sample is due to recombination rather than trapping, as saturation of deep level traps with increasing intensity would slow the decay of excitons [7]. Noting that the spot size of the probe is $\sim 100 \mu \mathrm{m}$ in diameter, comparison of the ratio of the decay components $\mathrm{C}_{2} / \mathrm{C}_{1}$ to the ratio of stripe size to window size suggests that the slow $\tau_{2}$ decay may represent the exciton lifetime in the LEO material, while the fast $\tau_{1}$ decay may be characteristic of the highly defective window region. Low temperature $\Delta \mathrm{R}$ studies of resonantly excited exciton dynamics in LEO metalorganic vapor phase epitaxy (MOVPE) material [6] and time-resolved photoluminescence (TRPL) studies of hydride vapor phase epitaxy (HVPE) material [8] yield free exciton lifetimes of 375 ps at $60 \mathrm{~K}$ and $295 \mathrm{ps}$ at $4 \mathrm{~K}$, respectively. These results are in good agreement with theoretical predictions of the radiative lifetime [9]. At room temperature the exciton lifetime is longer ( $720 \mathrm{ps})$ in our MOCVD-grown LEO material than that reported (530 ps) for the high quality HVPE sample, with the long lifetimes in both cases associated with a thermally induced increase in the center of mass kinetic energy of the excitons. While the $\tau_{1}$ of 55 ps in our LEO sample is consistent with decay times observed in more defective GaN films, it is important to note that TRPL measurements capable of discriminating between the window and high quality materials [10] yielded a dominant decay time of $130 \mathrm{ps}$ for both cases when excitation pulses of $4.64 \mathrm{eV}$ photon energy were employed.

The fact that $\Delta \mathrm{R}$ possesses a positive rise near zero time delay for excitation of free carriers at $357.5 \mathrm{~nm}$ or $347 \mathrm{~nm}$ is indicative of the rapid thermalization of the electrons through electron-electron scattering, which causes the distribution to be weighted toward energies below the center of the probe spectrum (equation 1). Since the results described above imply that saturation of defect-related traps does not play an important role in the exciton dynamics, we propose that the $\Delta \mathrm{R}$ decays for $357.5 \mathrm{~nm}$ excitation are primarily associated with the Coulomb capture of electron-hole pairs to form excitons. As the carrier density increases, the Coulomb interaction is more efficiently screened and the decay of the electron distribution is retarded. Viewed in this way, the decay time $\tau_{\mathrm{hm}} \sim 46$ ps at the lowest excitation density $\left(\sim 10^{18} \mathrm{~cm}^{-3}\right)$ becomes the exciton formation (capture) time at that carrier density.

Comparison of figures 2 and 3 shows that the $\Delta \mathrm{R}$ decays are much faster at a given carrier density for $347 \mathrm{~nm}$ excitation than for the $357.5 \mathrm{~nm}$ data. For the $347 \mathrm{~nm}$ case, the hot electron distribution cools primarily by transfer of energy to both the cold photoexcited hole distribution through electron-hole scattering, and to the lattice through electron-LO phonon scattering. At high excitation density (solid line, fig. 3) the slow decay suggests that the electron-LO phonon interaction is strongly screened, and the $\tau_{\mathrm{hm}}$ of $\sim 67 \mathrm{ps}$ represents the relaxation time associated with electron-hole scattering at this carrier density. The fact that the decay time is nearly halved when the carrier density is reduced by almost an order of magnitude (dashed line) implies that the partially screened electron-LO phonon interaction begins to play a role in the cooling process. This observation is supported by the data at short times (inset), for which the initial $1.25 \mathrm{ps}$ decay at this carrier density is attributed to the screened electron-LO phonon interaction. The absence of this initial decay when the carrier density is further lowered to $\sim 2 \times 10^{18} \mathrm{~cm}^{-3}$ suggests that the screening is attenuated enough that the initial relaxation 
process becomes faster than the temporal resolution of our experiment ( $200 \mathrm{fs})$, in agreement with the results of other researchers [11].

\section{CONCLUSIONS}

We have demonstrated that picosecond photoinduced reflectivity measurements can be used to obtain information about room temperature exciton and free carrier dynamics in LEO GaN. For resonant excitation of cold excitons, the $\Delta \mathrm{R}$ decay possesses a $720 \mathrm{ps}$ component attributed to the free exciton lifetime in this high quality material. For electrons with small excess energy $(<50 \mathrm{meV})$, the strong increase in the $\Delta \mathrm{R}$ decay rate with decreasing excitation density suggests that screening of the Coulomb interaction may play an important role in the processes of carrier relaxation and exciton formation. The faster decay times at a given carrier density observed for hot (>100 meV) electron relaxation are attributed to electron-hole scattering in conjunction with the screened electron-LO phonon interaction.

\section{ACKNOWLEDGEMENTS}

The authors thank NSF, ONR, and the State of Texas for partial support of this work at UT-Austin.

\section{REFERENCES}

1. S. Nakamura, M. Senoh, S. Nagahama, N. Iwasa, T. Yamada, T. Matsushita, H. Kiyoku, Y. Sugimoto, T. Kozaki, H. Umemoto, M. Sano, and K. Chocho, Appl. Phys. Lett. 72, 211 (1998).

2. D. Kapolnek, S. Keller, R. Vetury, R. Underwood, P. Kozodoy, S. DenBaars, and U. Misra, Appl. Phys. Lett. 71, 1204 (1997); T. Zheleva, O.-H. Nam, M. Bremser, and R. Davis, Appl. Phys. Lett. 71, 2472 (1997).

3. C. Sasaoka, H. Sunakawa, A. Kimura, M. Nido, A. Usui, and A. Sakai, J. Cryst. Growth 189/190, 61 (1998); P. Kozodoy, J. Ibbetson, H. Marchand, P. Fini, S. Keller, J. Speck, S. DenBaars, and U. Mishra, Appl. Phys. Lett. 73, 975 (1998).

4. D.H. Auston, S. McAfee, C.V. Shank, E.P. Ippen, and O. Teschke, Solid State Elec.21, 147 (1978).

5. J.S. Im, A. Moritz, F. Steuber, V. Härle, F. Scholz, and A. Hangleiter, Appl. Phys. Lett. 70, 631 (1997).

6. S. Hess, F. Walraet, R.A. Taylor, J.F. Ryan, B. Beaumont, and P. Gibart, Phys. Rev. $B$ 58, R15973 (1998).

7. H. Haag, B. Hönerlage, O. Briot, and R. L. Aulombard, Phys. Rev. B 60, 11624 (1999).

8. G.E. Bunea, W.D. Herzog, M.S. Ünlü, B.B. Goldberg, and R.J. Molnar, Appl. Phys. Lett. 75, 838 (1999).

9. Y. Toyozawa, Prog. Theor. Phys. Suppl.12, 111 (1959).

10. S.F. Chichibu, H. Marchand, M.S. Minsky, S. Keller, P.T. Fini, J.P. Ibbetson, S.B. Fleischer, J.S. Speck, J.E. Bowers, E. Hu, U.K. Mishra, S.P. DenBaars, T. Deguchi, T. Sota, and S. Nakamura, Appl. Phys. Lett. 74, 1460 (1999).

11. K.T. Tsen, D.K. Ferry, A. Botchkarev, B. Sverdlov, A. Salvador, and H. Morkoc, Appl. Phys. Lett. 71, 1852 (1997). 